\title{
Privacy-preserving Analytic Methods for Multisite Comparative Effectiveness and Patient-centered Outcomes Research
}

\author{
Sengwee Toh, ScD,* Susan Shetterly, MS † John D. Powers, MS, † and David Arterburn, MD, MPH $\ddagger$
}

\begin{abstract}
Background: For privacy and practical reasons, it is sometimes necessary to minimize sharing of individual-level information in multisite studies. However, individual-level information is often needed to perform more rigorous statistical analysis.
\end{abstract}

Objectives: To compare empirically 3 analytic methods for multisite studies that only require sharing of summary-level information to perform statistical analysis that have traditionally required access to detailed individual-level data from each site.

Research Design, Subjects, and Measures: We analyzed data from a 7-site study of bariatric surgery outcomes within the Scalable Partnering Network. We compared the long-term risk of rehospitalization between adjustable gastric banding and Roux-en-y gastric bypass procedures using a stratified analysis of propensity score (PS)-defined strata, a case-centered analysis of risk set data, and a meta-analysis of site-specific effect estimates. Their results were compared with the result from a pooled individual-level data analysis.

Results: The study included 1327 events (18.1\%) among 7342 patients. The adjusted hazard ratio was 0.71 (95\% CI, 0.59, 0.84) comparing adjustable gastric banding with Roux-en-y gastric bypass in the individual-level data analysis. The corresponding effect estimate was $0.70(0.59,0.83)$ in the PS-stratified analysis, 0.71 $(0.59,0.84)$ in the case-centered analysis, and $0.71(0.60,0.84)$ in both the fixed-effect and random-effects meta-analysis.

From the *Department of Population Medicine, Harvard Medical School and Harvard Pilgrim Health Care Institute, Boston, MA; †Institute for Health Research, Kaiser Permanente Colorado, Denver, CO; and $\ddagger$ Group Health Research Institute, Seattle, WA.

Supported by grant number 1R01HS019912 from the Agency for Healthcare Research and Quality. The content is solely the responsibility of the authors and does not necessarily represent the official views of the Agency for Healthcare Research and Quality.

The authors declare no conflict of interest.

Reprints: Sengwee Toh, ScD, Department of Population Medicine, Harvard Medical School and Harvard Pilgrim Health Care Institute, 133 Brookline Ave, 6th Floor, Boston, MA 02215. E-mail: darrentoh@post. harvard.edu.

Supplemental Digital Content is available for this article. Direct URL citations appear in the printed text and are provided in the HTML and PDF versions of this article on the journal's Website, www.lww-medical care.com.

Copyright (C 2014 by Lippincott Williams \& Wilkins

ISSN: 0025-7079/14/5207-0664
Conclusions: In this empirical study, PS-stratified analysis, casecentered analysis, and meta-analysis produced results that are identical or highly comparable with the result from a pooled individual-level data analysis. These methods have the potential to be viable analytic alternatives when sharing of individual-level information is not feasible or not preferred in multisite studies.

Key Words: comparative effectiveness research, patient-centered outcomes research, distributed research networks, confounding

(Med Care 2014;52: 664-668)

$M$ ultisite data networks support a wide range of comparative effectiveness research (CER) and patient-centered outcomes research (PCOR) activities. They allow studying of research questions that cannot be addressed by individual data sources and increase the generalizability of study findings. The Patient-Centered Outcomes Research Institute recently committed $\$ 100$ million to create the National Patient-Centered Clinical Research Network (PCORnet). The initiative will create 11 Clinical Data Research Network, 18 Patient-Powered Research Networks, and a coordinating center. ${ }^{1}$ It joins other innovative national initiatives that are building distributed data networks to generate timely and actionable evidence, such as the Mini-Sentinel program ${ }^{2,3}$ and the NIH Health Care Systems Research Collaboratory.,

Minimizing sharing of sensitive or highly granular patient information is key to the success and sustainability of multisite collaboratives, as sharing of such information may introduce concerns about patient privacy, data security, and unapproved use of data after they leave the data partners' firewalls. Traditionally, it has often been necessary to share detailed individuallevel information to adjust for confounding and other biases inherent in observational CER or PCOR studies.

Newer methods that require only summary-level information while achieving similar level of statistical sophistication have emerged from other fields (eg, medical product safety surveillance and bioinformatics) in recent years, ${ }^{6-10}$ but they have not been systematically assessed for CER or PCOR. We have previously described the theoretical properties of some of these methods and how they may be applied in CER. ${ }^{11}$ In this study, we implemented 3 of these methods in a real-world CER study to provide empirical evidence of their validity compared with a pooled individuallevel data analysis. 


\section{METHODS}

\section{Data Source}

This study was conducted within the Scalable Partnering Network (SPAN) for CER (http://www.span-network.org/), which included 10 demographically and geographically distributed health care systems in the United States. The SPAN network was funded by the Agency for Healthcare Research and Quality (AHRQ) to develop the informatics platform, governance processes, and scientific expertise to conduct research in a distributed health network. All sites participating in the SPAN network use electronic health records in routine care delivery, and have transformed their source data into a common data model. This study was approved by the Kaiser Permanente Colorado (KPCO) Institutional Review Board (IRB), and the requirement for informed consent was waived. Participating sites either ceded IRB oversight to the KPCO IRB or obtained IRB approval from their sites' IRBs.

\section{Study Setting}

This methods project was part of a larger study designed to examine the short-term and long-term effects of 2 of the most common bariatric surgical procedures, the laparoscopic adjustable gastric band (AGB) and the laparoscopic Roux-en-y gastric bypass (RYGB) procedure. As part of the main study, all participating sites had created and transferred a limited deidentified individual-level dataset to the lead team at KPCO. Individuals were eligible if they underwent laparoscopic AGB or RYGB surgery between January 1, 2005 and December 31, 2009, were aged 21 years and above at the time of initial bariatric surgery, and were enrolled in the health system during the year before surgery. They were excluded if all recorded body mass indexes were $<35 \mathrm{~kg} / \mathrm{m}^{2}$ during the presurgery year. The outcome of interest was the risk of rehospitalization within the first 2 years after the discharge date of the initial surgery.

For the purposes of this methods study, we created a distributed data system internally at KPCO by storing the site-specific individual-level datasets at separate locations. We analyzed data from 7 of the 10 sites participating in the main study, excluding 3 sites with $<20$ AGB-treated patients. The threshold was chosen so that minimally adjusted sitespecific analysis with only age and sex adjustment would be possible. ${ }^{12,13}$

\section{Pooled Individual-level Data Analysis (Referent Analysis)}

Using the pooled individual-level information from the 7 participating sites, we fit a Cox proportional hazards model with site and site-specific propensity score (PS) quintile as stratification variables to estimate the adjusted hazard ratio of rehospitalization comparing AGB with RYGB. The sitespecific PSs were the probabilities of undergoing the AGB procedure, estimated within each site using a logistic regression model that included all the covariates listed in Table 1 as independent variables. These covariatesascertained during the 365-day period before the initial surgery date-were selected based on scientific and clinical
TABLE 1. Patient Characteristics, By Bariatric Procedure Type

\begin{tabular}{lcc}
\hline & \multicolumn{2}{c}{ N (\%)* } \\
\cline { 2 - 3 } Characteristics & $\begin{array}{c}\text { Adjustable Gastric } \\
\text { Band (n = 1550) }\end{array}$ & $\begin{array}{c}\text { Roux-en-y Gastric } \\
\text { Bypass }(\mathbf{n}=\mathbf{5 7 9 2})\end{array}$ \\
\hline Mean age (SD) & $46.7(11.2)$ & $45.7(10.7)$ \\
Age $>65 \mathrm{y}$ & $76(4.9)$ & $141(2.4)$ \\
Female sex & $1266(81.7)$ & $4823(83.3)$
\end{tabular}

Race/ethnicity

Black or African

American

White

Hispanic

Other

Unknown

Year of procedure

2005

2006

2007

2008

2009

Mean baseline body

mass index (SD)

Baseline body mass

index category

30-34.9

35-39.9

40-49.9

$\geq 50$

Mean Charlson

comorbidity score

(SD)

Comorbidity

Asthma

Atrial fibrillation

Smoking status

Mean blood pressure (SD)

$137(8.8)$

$522(9.0)$

1130 (72.9)

$142(9.2)$

$62(4.0)$

$79(5.1)$

$3840(66.3)$

$769(13.3)$

$280(4.8)$

381 (6.6)

$0(0.0)$

134 (8.6)

417 (26.9)

552 (35.6)

$447(28.8)$

$42.5(5.7)$

$408(7.0)$

$980(16.9)$

$1352(23.3)$

$1515(26.2)$

$1537(26.5)$

$44.5(6.7)$

$96(6.2)$

$480(31.0)$

$813(52.4)$

$161(10.4)$

$1.0(1.2)$

$174(3.0)$

$1410(24.3)$

$3126(54.0)$

$1082(18.7)$

$1.1(1.2)$

$\begin{array}{cc}364(23.5) & 1588(27.4) \\ 36(2.3) & 97(1.7) \\ 39(2.5) & 123(2.1) \\ & \\ 95(6.1) & 291(5.0) \\ 712(45.9) & 2522(43.5) \\ 888(57.3) & 3467(59.9) \\ 388(25.0) & 1967(34.0) \\ 15(1.0) & 42(0.7) \\ & \\ 12(0.8) & 36(0.6) \\ 526(33.9) & 2199(38.0) \\ & \\ 596(38.5) & 2530(43.7) \\ 15(1.0) & 67(1.2) \\ & \\ 60(3.9) & 185(3.2) \\ 22(1.4) & 51(0.9) \\ & \\ 635(41.0) & 2566(44.3) \\ 724(46.7) & 2971(51.3) \\ 191(12.3) & 255(4.4) \\ & \\ 27.0(15.2) & 129.3(15.5) \\ 77.6(9.6) & 78.5(10.2) \\ & \\ 0.29(3.0) & 0.28(3.5) \\ 41(2.7) & 151(2.6) \\ & \\ 24(1.5) & 128(2.2) \\ 101(6.5) & 522(7.5) \\ 1122(72.4) & \\ 380(24.5) & \\ & \\ & \\ & \\ & \\ & \\ & \\ & \end{array}$

Length of stay associated

with procedure

Insurance type
Congestive heart

failure

Coronary artery

disease

Hyperlipidemia

Hypertension

Diabetes

Deep vein

thrombosis

Pulmonary embolism

Gastroesophageal

reflux disease

Sleep apnea

Assistive walking

device

Oxygen use

Anticoagulant use

Ever smoker

Never smoker

Missing

Systolic

Mean (SD)

$>2 \mathrm{~d}$

Medicaid

Medicare

Commercial

$380(24.5)$

$3(2.1)$

$291(5.0)$

$67(34.0)$

$2(0.7)$

$36(0.6)$

$2530(43.7)$

$67(1.2)$

$185(3.2)$

$1(0.9)$

$566(44.3)$

$971(51.3)$

5 (4.4)

$9.3(15.5)$

$8.5(10.2)$

$.28(3.5)$

$28(2.2)$

$172(89.3)$

Other

*Percent within each treatment group. 
considerations. We considered this pooled individual-level data analysis our referent analysis.

\section{Applications of 3 Methods That Analyze Summary-level Information}

We examined 3 analytic methods that do not require sharing individual-level information to estimate the association between bariatric surgery and rehospitalization, while adjusting for multiple potential confounders. Each of these methods (described below; technical detail provided in the web appendix, Supplemental Digital Content 1, http://links. lww.com/MLR/A727) requires different types of summarylevel information from the participating sites. We compared the results from these methods with the result from the pooled individual-level analysis.

\section{PS Stratification}

We performed an analysis stratified by site and sitespecific PS quintile. Each site first independently estimated the PSs as described above, then created a dataset that contained only 5 records, each defined by a PS stratum. Each record included the number of follow-up person-times and outcome events in each treatment group. ${ }^{11}$ Upon receiving all the summary-level datasets, the lead team used the Mantel-Haenszel approach to obtain the overall effect estimate and $95 \%$ confidence interval (CI). ${ }^{14}$

\section{Case-centered Analysis}

In this approach, the participating sites also estimated the site-specific PSs as described above. They then created a site-specific dataset that included 1 record per risk set (defined below). The dataset had as many records as the number of cases (ie, patients with an outcome event) because each risk set is anchored by a case. The risk set comprised the case and patients who were at risk of the outcome at the time of event occurrence and from the same PS stratum as the case. Each record in the dataset contained a binary variable indicating whether the case underwent AGB or RYGB and the log odds of the proportion of AGB-treated patients in the risk set. Using these records from all sites, the lead team fit a logistic regression model with the binary exposure variable as the dependent variable and the $\log$ odds as the independent variable (specified as an offset). Fireman et $\mathrm{al}^{8}$ have shown that such a model maximizes the same likelihood as a Cox model fit using individual-level data, and both yield the same parameter estimates.

\section{Meta-Analysis}

We performed an inverse variance-weighted metaanalysis. ${ }^{15,16}$ Each site first fit an individual-level Cox proportional hazards model with site-specific PS quintile as a stratification variable. It then transferred its site-specific adjusted hazard ratio and $95 \% \mathrm{CI}$ to the lead team, who used both fixed-effect and random-effects models to pool all the site-specific estimates.

\section{RESULTS}

Table 1 shows the distributions of the potential confounders by treatment group. The site-specific HRs, adjusted for these potential confounders by PS stratification, were all $<1$, with some variation in the magnitude of the effect size (Table 2). The $P$-value for the test of homogeneity was 0.52 . The overall adjusted HR was 0.71 (95\% CI, 0.59, 0.84) from the referent analysis using the pooled individual-level data from the 7 participating sites. The 3 methods that only require summary-level information produced similar results (Table 3); in fact, the result from the case-centered analysis was identical to the result from the referent analysis. The fixed-effect and random-effects meta-analysis produced the same result.

\section{DISCUSSION}

In this study, we provide empirical evidence that shows that it is possible to perform relatively sophisticated statistical analysis using summary-level data in multisite CER studies. In this particular CER example, the 3 methods produced results that were identical or highly comparable with that obtained from the pooled individual-level data analysis.

A fully functional distributed research network should have the capability to perform analyses that are both feasible and valid. However, it is not uncommon to encounter situations where sharing of individual-level information is not possible or preferred in multicenter studies. It is therefore important to develop alternative analytic methods like

TABLE 2. Results From Individual-level Data Analysis, By Site

\begin{tabular}{|c|c|c|c|c|c|c|c|}
\hline \multirow[b]{2}{*}{ Site } & \multirow[b]{2}{*}{ Size } & \multicolumn{2}{|c|}{ Band } & \multicolumn{2}{|c|}{ Bypass } & \multirow[b]{2}{*}{$\begin{array}{c}\text { Adjusted Hazard } \\
\text { Ratio }^{\ddagger}\end{array}$} & \multirow[b]{2}{*}{$\begin{array}{c}\text { 95\% Confidence } \\
\text { Interval }\end{array}$} \\
\hline & & $\begin{array}{l}\text { Patients } \\
\text { N }(\%)^{*}\end{array}$ & $\begin{array}{l}\text { Events } \\
\mathbf{N}(\%)^{\dagger} \\
\end{array}$ & $\begin{array}{l}\text { Patients } \\
\text { N }(\%)^{*}\end{array}$ & $\begin{array}{l}\text { Events } \\
\mathbf{N}(\%)^{\dagger}\end{array}$ & & \\
\hline Site 2 & 752 & $123(16.4)$ & $16(13.0)$ & $629(83.6)$ & $145(23.1)$ & 0.65 & $0.37,1.15$ \\
\hline Site 3 & 265 & $107(40.4)$ & $16(15.0)$ & $158(59.6)$ & $35(22.2)$ & 0.52 & $0.26,1.04$ \\
\hline Site 4 & 636 & $112(17.6)$ & $10(8.9)$ & $524(82.4)$ & 93 (17.7) & 0.72 & $0.35,1.50$ \\
\hline Site 5 & 852 & 109 (12.8) & $15(13.8)$ & $743(87.2)$ & $186(25.0)$ & 0.82 & $0.46,1.48$ \\
\hline
\end{tabular}

*Percent of patients with treatment at a site.

†ercent of patients with event within each treatment group at a site.

*From site-specific Cox proportional hazards model stratified on propensity score quintile. 
TABLE 3. Results From Adjusted Analysis, By Method

\begin{tabular}{lcc}
\hline Methods & Point Estimate & $\mathbf{9 5 \%}$ CI \\
\hline Individual-level data analysis & 0.71 & $0.59,0.84$ \\
PS-stratified analysis & 0.70 & $0.59,0.83$ \\
Case-centered analysis & 0.71 & $0.59,0.84$ \\
Fixed-effect meta-analysis & 0.71 & $0.60,0.84$ \\
Random-effects meta-analysis & 0.71 & $0.60,0.84$ \\
\hline
\end{tabular}

CI indicates confidence interval; PS, propensity score.

the ones assessed in this study. Empirical examples have the ability to enhance understanding of these methods and promote discussions on the potential utility of such methods. The biggest strength of these methods is that they require only summary-level information to perform relatively sophisticated statistical analysis traditionally afforded by having individual-level information. When used in combination with confounder summary scores (eg, PSs or disease risk scores), these methods allow one to adjust for a large number of confounders with little concern about sharing highly granular and potentially identifiable information. ${ }^{7,11}$ We have previously discussed the pros and cons of using confounder summary scores in distributed research networks. ${ }^{11}$

A major strength of the current study is the availability of a referent analysis against which the 3 methods could be reasonably compared. We used a pooled individual-level data analysis as our reference because traditionally it represents the best possible analysis one could perform in a multicenter study. To the best of our knowledge, this is the first study to empirically compare these methods to a pooled individual-level data analysis in the context of CER.

In contrast, our studies are not without limitations. We conducted the analysis in a "simulated" distributed data system, so we were not able to assess the logistical efficiency of these methods when applied in actual distributed systems. In principle, all 3 methods can be done using the distributed program developed by the lead team. However, some may require more analytic support from the participating sites than the others if unexpected errors or warnings occur when executing the distributed program.

Removing sites with a small sample size from our analysis might have artificially improved the comparability of results across methods. It has been shown in simulation studies that when the sample size is small, certain site-specific analyses may not be feasible or may produce unreliable estimates, leading to different results across methods. ${ }^{17} \mathrm{We}$ stratified our analysis on site-specific PS quintile and did not assess whether these methods would provide comparable results if we stratified more or less finely, or matched on PSs. However, the theoretical properties of the case-centered analysis would predict that its results would be the same as the results from the pooled individual-level data analysis. Finally, our findings may not be generalizable to other study settings, for example, research networks that do not use a common data model.

In conclusion, we have provided empirical evidence that the PS-stratified analysis, the case-centered analysis, and the meta-analysis can produce results that are identical or highly comparable with the result obtained from a pooled individual-level data analysis. These methods have the potential to be viable analytic alternatives when sharing of individual-level information is not feasible or not preferred in multisite CER or PCOR studies. However, more simulations and empirical studies are needed to better understand their performance in various study settings.

\section{ACKNOWLEDGMENTS}

The authors thank the programmer/analysts from the following SPAN sites for providing data for this project: Essentia Institute for Rural Health (EIRH), Group Health Research Institute (GHRI), Harvard Pilgrim Health Care (HPHC), HealthPartners Institute for Education and Research (HPIER), Kaiser Permanente Northern California (KPNC), Kaiser Permanente Colorado (KPCO), and Kaiser Permanente Hawaii (KPHI).

The authors are indebted to the following SPAN Investigators for the collaborations that made this study possible: KPCO [Matthew F. Daley, MD (SPAN PI); Elizabeth A. Bayliss, MD, MSPH; Ella Lyons, MS], EIRH (Thomas E. Elliott, MD), HPIER (Pamala A. Pawloski, PharmD), KPHI (Cynthia Nakasato, MD; Rebecca Williams, DrPH, MPH; Vinutha Vijayadeva, PhD), and KPNC (Lisa Herrinton, PhD).

Finally, the authors would like to thank Stanley Xu, $P h D$ (KPCO) and Lingling Li, PhD (HPHC) for reviewing the appendix (Supplemental Digital Content 1, http://links. lww.com/MLR/A727).

\section{REFERENCES}

1. Patient-Centered Outcomes Research Institute. PCORnet: The National Patient-Centered Clinical Research Network. 2014. Available at: http:// www.pcori.org/funding-opportunities/pcornet-national-patient-centeredclinical-research-network/. Accessed February 5, 2014.

2. Behrman RE, Benner JS, Brown JS, et al. Developing the Sentinel System - a national resource for evidence development. $N$ Engl J Med. 2011;364:498-499.

3. Platt R, Carnahan RM, Brown JS, et al. The US Food and Drug Administration's Mini-Sentinel program: status and direction. Pharmacoepidemiol Drug Saf. 2012;21(suppl 1):1-8.

4. National Institutes of Health. NIH Health Care Systems Research Collaboratory. Available at: https://www.nihcollaboratory.org/Pages/ default.aspx. Accessed February 9, 2014.

5. Califf RM, Platt R. Embedding cardiovascular research into practice. JAMA. 2013;310:2037-2038.

6. Rassen JA, Moran J, Toh D, et al. Evaluating strategies for data sharing and analyses in distributed data settings. 2013. Available at: http:// www.mini-sentinel.org/work_products/Statistical_Methods/Mini-Sentinel_ Methods_Evaluating-Strategies-for-Data-Sharing-and-Analyses.pdf. Accessed March 28, 2013.

7. Rassen JA, Solomon DH, Curtis JR, et al. Privacy-maintaining propensity score-based pooling of multiple databases applied to a study of biologics. Med Care. 2010;48:S83-S89.

8. Fireman B, Lee J, Lewis N, et al. Influenza vaccination and mortality: differentiating vaccine effects from bias. Am J Epidemiol. 2009;170: $650-656$.

9. Karr AF, Lin X, Sanil AP, et al. Secure regression on distributed databases. J Comput Graph Stat. 2005;14:263-279.

10. Wu Y, Jiang X, Kim J, et al. Grid Binary LOgistic REgression (GLORE): building shared models without sharing data. J Am Med Inform Assoc. 2012;19:758-764. 
11. Toh S, Gagne JJ, Rassen JA, et al. Confounding adjustment in comparative effectiveness research conducted within distributed research networks. Med Care. 2013;51:S4-S10.

12. Peduzzi P, Concato J, Kemper E, et al. A simulation study of the number of events per variable in logistic regression analysis. J Clin Epidemiol. 1996;49:1373-1379.

13. Cepeda MS, Boston R, Farrar JT, et al. Comparison of logistic regression versus propensity score when the number of events is low and there are multiple confounders. Am J Epidemiol. 2003;158:280-287.

14. Rothman KJ, Greenland S, Lash TL. Modern Epidemiology. Philadelphia, PA: Lippincott Williams \& Wilkins; 2008.
15. Deeks JJ, Higgins JPT, Altman DG. Chapter 9: Analysing data and undertaking meta-analyses. In: Higgins JPT, Green S, eds. Cochrane Handbook for Systematic Reviews of Interventions Version 501 [updated September 2008]. Chichester, West Sussex: The Cochrane Collaboration; 2008.

16. DerSimonian R, Laird N. Meta-analysis in clinical trials. Control Clin Trials. 1986;7:177-188.

17. Toh S, Reichman ME, Houstoun $\mathrm{M}$, et al. Multivariable confounding adjustment in distributed data networks without sharing of patient-level data. Pharmacoepidemiol Drug Saf. 2013;22: $1171-1177$ 